\title{
Thermal Induced Structural Conductivity in LPCVD Polysilicon Film on Silicon Nitride/SiO Capped (100) Silicon
}

\author{
Shobha Kanta Lamichhane ${ }^{1}$ and Jamil Akhtar ${ }^{2}$ \\ ${ }^{1}$ Tribhuvan University, Prithwi Narayan Campus, Pokhara, Nepal \\ ${ }^{2}$ Microelectronics Technology Group, CEERI, Pilani - 333031, India \\ e-mail:sklamichhane@hotmail.com
}

\begin{abstract}
Polysilicon (PS) grains are clustered in an order in the presence of thermal doping of boron in low pressure chemical vapour deposition (LPCVD). PS layer is lying on silicon nitride/silicon dioxide bed over (100) silicon substrate. The doped PS at different temperatures has been analyzed for the grain size and the shape of the clusters, employing non-contact mode atomic force microscopy (AFM). The grain size of the PS remains intact without a significant change with increasing doping temperature. A substantial increase in the cluster size and its density of the grains has been observed. The cluster formation mechanism induced by thermal variation is discussed in the context of recorded AFM images. The clusters lead to PS rings comprising of grains of the size of $100 \mathrm{~nm}$.
\end{abstract}

Keywords: doping, polisilicon, nanophysics, grainsize, AFM, MEMS, LPCVD, SOI, contactmode

\section{Introduction}

Polycrystalline silicon (PS), being a piezoresistive material and having excellent compatibility with matured silicon technology, has large-scale potential in the emerging field of micro-electro-mechanical systems (MEMS) Tilke et al. 2002. It has super high temperature characteristics, which are desirable for harsh environment applications such as turbine engine and rocket propulsion system, etc and hence one of the materials at the forefront of process development Tilke et al. 2002, McConn et al. 2001. Formation of high-quality PS on low cost substrates has important applications in the development of thin film solar cells, transistors, image sensors, etc. PS is most widely used as a structural material in current micro devices. In the field of metal oxide semiconductor (MOS) based devices and circuits, PS has been a well established material for the gate Tai et al. 1988, Change \& see 1996. More recently PS has been identified a promising material for realizing nanostructures embedded into silicon on insulation (SOI) structures. Owing to its increasing applications, efforts are being made to characterize the PS films particularly deposited on insulating bed using LPCVD techniques Maseeh \& Senturia 1990.
Thin film silicon dioxide has a compressive internal stress, less stiff than other thin film materials, has unique electrical properties and used as a mechanical material in high sensitivity applications. Silicon dioxide, with its low thermal conductivity, is a natural thermal insulator, a property which has been exploited for the production of integrated thermal detector. In MEMS, it is used to electrically isolate components and has been used in some recent applications as a structural material Kalainathan et al. 1990.

Silicon nitride is a material that is employed in a variety of applications. Since it does not react well with many etching solutions, silicon nitride is often used to prevent impurity diffusion and ionic contamination. One of the unfortunate properties of silicon nitride is that it is not as good insulator as silicon dioxide. With a bandgap $40 \%$ smaller than $\mathrm{SiO}_{2}$, the electrical isolation provided by silicon nitride is significantly less than that of silicon dioxide. $\mathrm{Si}_{3} \mathrm{~N}_{4}$ forms a low energy barrier towards 
silicon, which facilitates the injection of holes into the substrate. Due to these concerns, some designers like to form most of an insulator with $\mathrm{SiO}_{2}$ and then seal its surface with Si3N4 Tranco et al. 2001.

Control of the electrical conductivity of the PS layers is carried out using doping of compatible extrensic element such as boron for $\mathrm{p}$-type. However doping distribution of the extrensic element does not follow the way it is characterized in single crystalline silicon. Effect of grain boundaries on the doping distribution in the PS layers has been studied with findings related to doping species confining to the edges of the grains Karnik \& Hatalis 2003. Recently interest has been grown for analyzing the grain size of the PS films and its impact on the device characteristics Pacellia et al. 1999. Thermal conductivity is another parameter that attend much importance due to its inherent association with the grain size of the PS layer.

In the present work, LPCVD PS film has been realized on the bed of composite layers of silicon nitride and silicon dioxide over (100) silicon substrate. The PS film is doped using thermal diffusion of boron at four different temperatures to adjust the sheet resistivity (SR). The thermally treated PS film is analyzed for its topological details using non-contact mode AFM in the atmospheric pressure. The grain size of the PS film has been analyzed with varying temperatures in the presence of boron doping.

The details of the experiment carried out are presented in the next section. The images of the grains and the clusters observed using AFM are analyzed with relevant discussion. The work is concluded at the end.

\section{Materials and Methods}

Starting from a p-type (100) silicon wafer, thermal oxide was grown for a thickness of $0.5 \mu \mathrm{m}$ using conventional dry-wet-dry change see 1996 procedure in a quartz furnace. Silicon nitride was then deposited over the oxide using LPCVD, at $780^{\circ} \mathrm{C}$, for a thickness of $0.15 \mu \mathrm{m}$. The thickness of the composite layers was crucial to ensure for the strain balance at the interface of the silicon oxide and silicon nitride Maseeh \& Senturia 1990.
PS was deposited on the silicon nitride bed using LPCVD at $620^{\circ} \mathrm{C}$ in a furnace with a Silane $\left(\mathrm{SiH}_{4}\right)$ flow rate of $50 \mathrm{~cm}^{3} / \mathrm{min}$ and a process pressure of 0.3 torr. The thickness of the PS film was measured of $0.5 \mu \mathrm{m}$ using Nanospec Optical Spectrophotometer. Boron doping of the PS film was carried out in a furnace at four different temperatures of $920^{\circ} \mathrm{C}, 970^{\circ} \mathrm{C}, 1020^{\circ} \mathrm{C}$ and $1070^{\circ} \mathrm{C}$, for $40 \mathrm{~min}$ in nitrogen ambient with conventional flow rate. The borosilicate glass (BSG) invariably deposited on the PS, was etched out in buffered HF. The wafers with doped PS film at four different temperatures were oxidized at $850^{\circ} \mathrm{C}$ for 10 minutes in steam. After buffered HF treatment, all the four wafers were annealed at $920^{\circ} \mathrm{C}$ for 30 minutes in nitrogen ambient.

The AFM of thermo-microscope (TM), USA, auto probe electronic module, model no 1000 was used for taking micrographs. A silicon probe of radius $10 \mathrm{~nm}$ was used in contact mode. Repeated scans were conducted to get consistent images. In the following, surface morphology observed by AFM is presented.

\section{Results and Discussion}

PS is deposited and doped using different techniques, which affect the microstructure (i.e., grain size and shape and their concentration). Its properties strongly depend on deposition process and deposition temperature. Thin PS film is deposited on silicon substrate may have residual stress. During deposition, residual stress can be controlled entirely by varying deposition pressure and temperature. Thus mechanical property of PS film depends greatly upon the process used in deposition. In this study attention is being taken appreciably.

The PS films exhibit preferential grain orientations should vary with temperature. Since an ideal film does not exhibit orientation dependence for its mechanical properties. Researchers have found that depositing films at $590^{\circ} \mathrm{C}$, which is the transition point between polycrystalline and amorphous silicon, is an effective method of producing an isotropic film of PS. At this temperature amorphous silicon will recrystalize during annealing and produces film with nearly uniform Young's modulus. 
S.K. Lamichhane \& Jamil Akhtar/ Thermal Induced Structural ......

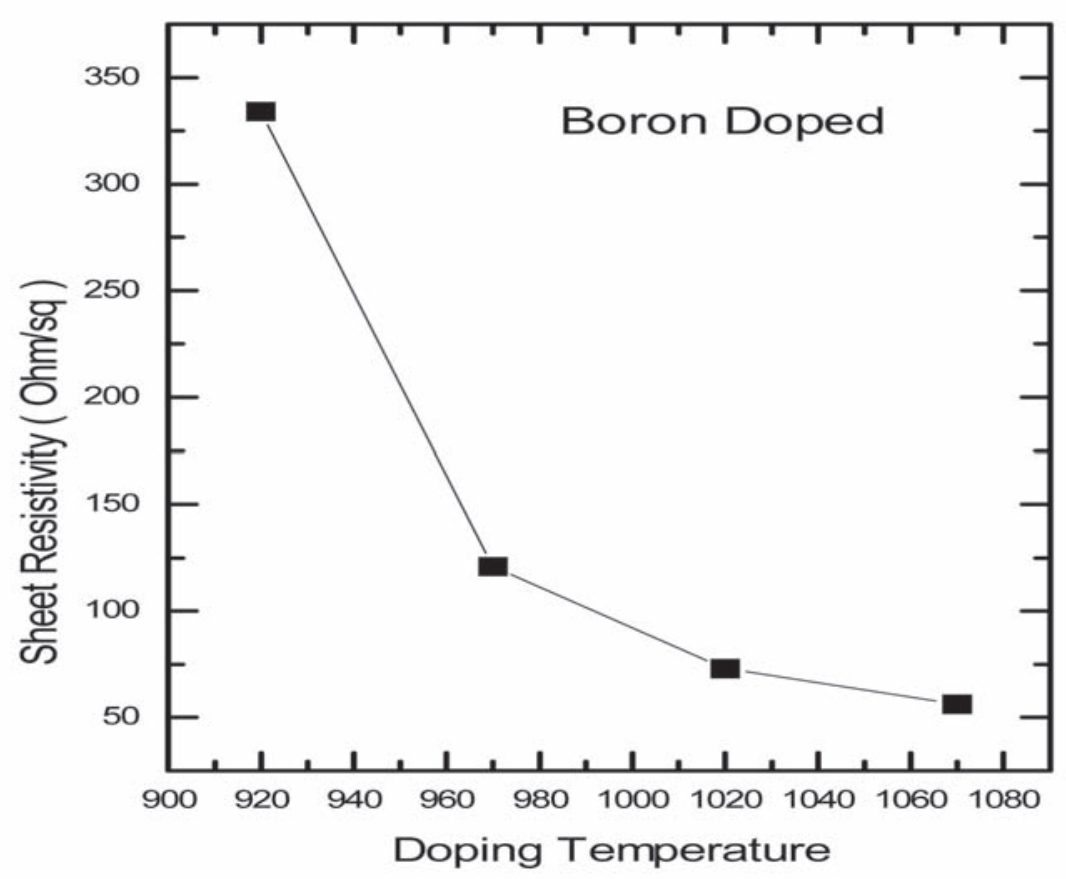

Fig. 1. sheet resistivity variation of LPCVD polysilicon film doped with boron at different doping temperatures

The PS films, doped separately with boron at $920^{\circ} \mathrm{C}$, $970^{\circ} \mathrm{C}, 1020^{\circ} \mathrm{C}$ and $1070^{\circ} \mathrm{C}$ were electrically characterized by measuring the sheet resistivity (SR) using four point probe method. Fig.1 shows the variation of the sheet resistivity of the PS layers with varying temperature. As a thumb rule, PS sheet resistivity doubles that of crystalline silicon at equivalent doping conditions change \& see 1996. The trend of the SR variation and the numerical values ensure the complete removal of the borosilicate glass from the PS. Beyond critical

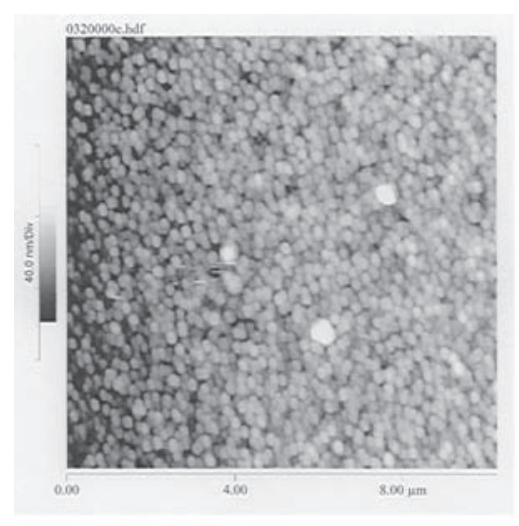

(a) temperature, PS film starts crystallization and form special kind of geometrical structure in the form of grains. The sizes of grains are no more than film thickness. AFM in non-contact mode was employed to analyse the grains of the PS layer. The AFM micrographs of the PS films doped at $920^{\circ} \mathrm{C}$ and $1070^{\circ} \mathrm{C}$ have been shown in the Fig. 2 and Fig. 3 respectively. The AFM micrographs confirm the polycrystalline nature of the doped film. The doping temperature does not affect the grain size of the PS as is evident from Fig. 2(a) and Fig. 3(a).

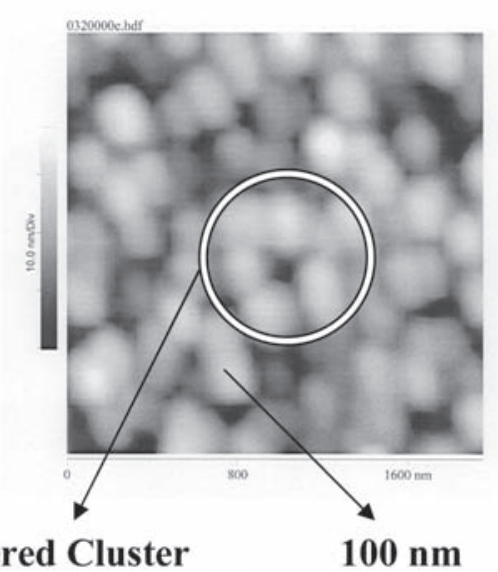

(b)

Fig. 2. AFM micrograph of polysilicon grains doped at 920 degree C. (a) Scan area of $10.0 \mu \mathrm{m} \times 10.0 \mu \mathrm{m}$, (b) scan area of $1.6 \mu \mathrm{m} \times 1.6 \mu \mathrm{m}$ 


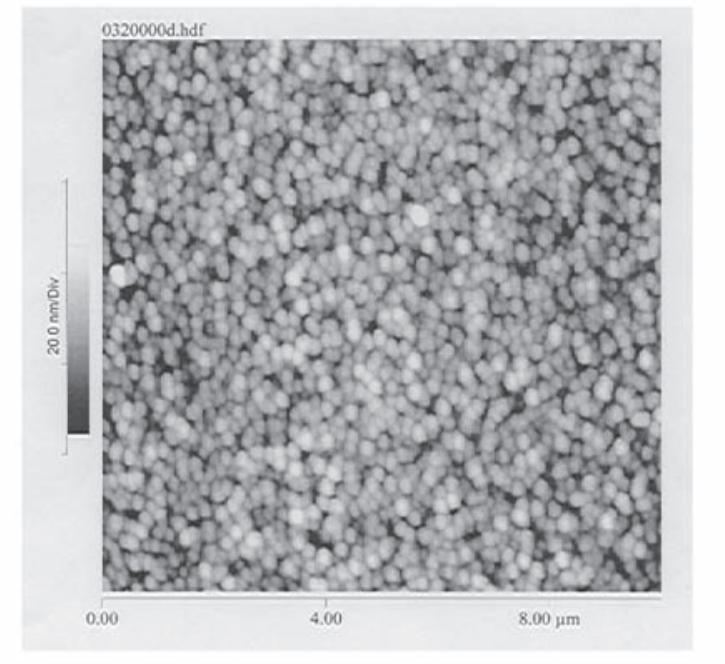

\section{Ordered Cluster}

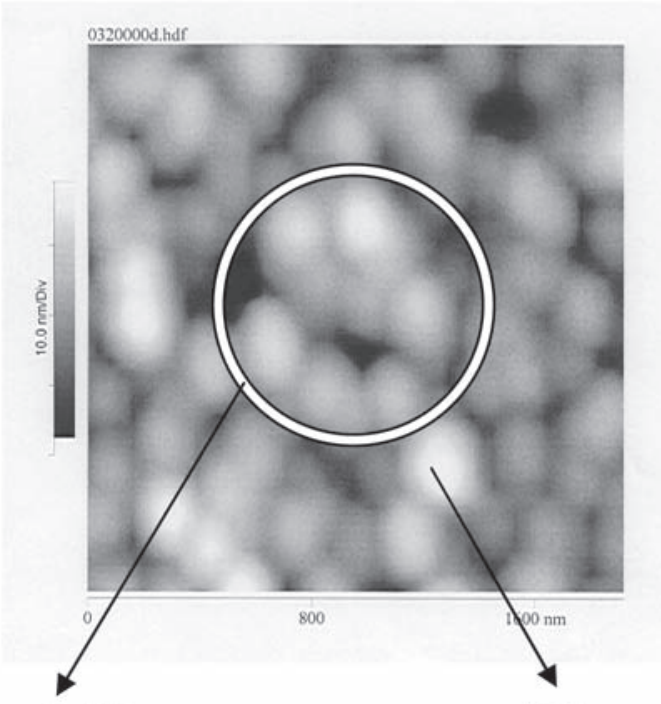

$100 \mathrm{~nm}$

(a)

(b)

Fig. 3. AFM micrograph of polysilicon grains doped at 1070 degree C. (a) scan area of $10.0 \mu \mathrm{m} \times 10.0 \mu \mathrm{m}$, scan area of $1.6 \mu \mathrm{m} \times 1.6 \mu \mathrm{m}$

Careful examining of the micrographs indicates, aligning of the grains in the shape of rings. The density of the grains in one ring grows further with increasing doping temperature. Fig. 2 (b) and 3(b) show the enlarged version of the micrographs for PS layers doped at $920^{\circ} \mathrm{C}$ and $1070^{\circ} \mathrm{C}$ respectively. In both the images, the average size of $100 \mathrm{~nm}$ of the PS grain confirms grain size invariance with doping temperature. Variation of grain size of the PS film with varying temperature has been considered a common phenomenon Ahmad et al. 2002. However, in the present case stability in the grain size with varying temperature and in the presence of boron doping attracts attention. To explain such results focus is given to fundamental understanding of invariance of grain structure with annealing.

As boron is doped into silicon there is deficiency of one electron in comparison with pure silicon. The valence band of doped silicon is therefore no longer completely full. Thus, under the influence of thermal energy electron can change its state and current can flow. The mechanism of this type of conductivity is ptype. Consider an electronic state of wave vector ' $k$ ' near the edge of the valence band, and with the energy less than that associated with the band edge. The energy difference, $\Delta E$, is

$$
\Delta E=\frac{1}{2 m_{v}}\left[\hbar\left(k-k_{0}\right)\right]^{2}
$$

When an electron is in a state near the edge of the band, it does not like free electron. Because of the strong interaction between the electron wave and the lattice vibration, force is developed which causes it to accelerate in the opposite direction. The movement of an electron on the top of the almost full band is equivalent to the movement of a positive hole at the bottom of the band. Hence, a hole is liberated by thermal agitation and delocalized in the lattice. In this way, the existence of p-type conductivity $(\sigma)$ is an essential feature in physics and technology. The magnitude of conductivity is

$$
\sigma=n|e| \mu_{h}=2\left(\frac{m k_{B} T}{2 \pi \hbar^{2}}\right)^{\frac{3}{2}} e^{-\left(\frac{E_{g}}{2 k_{B} T}\right)} \cdot|e| \mu_{h}
$$

Symbols have usual meaning.

This means in PS film, holes are responsible for transport of heat as well as conduction. As we know from basic physics, above absolute zero temperature, there is gradual increase in the amplitude of atomic vibration that influence lattice distortion. As a result, transverse and longitudinal phonons are generated 
during the process. Part of these phonons is responsible for thermal conductivity where as remarkable fraction of phonons should be involved to electrical conductivity.

It is recognize that Fermi level marks the dividing line, in energy, between whether state is more probably occupied or more probably empty. The scattering of electron by vibrating ions in a solid at a temperature $T$, the energy transfer between the electron and the ion is in the order of $k_{B} T$. If an electron is in a state with energy below the Fermi energy, the probability that such a state is occupied is essentially one. The probability of an unoccupied state with same energy is essentially zero. There are many such electrons since the probability that such a state is occupied is essentially one and the probability of an unoccupied state with energy close to this value is essentially zero. Because, Pauli Exclusion Principle is prohibits more than a couple of electron having opposite spin form occupying a state. Thus there is no available state as well as energy exchange between electrons inside the Fermi sphere since all available electron states are already occupied. This may be the probable reason why grain size remains invariance with thermal agitation in boron doped polysilicon film.

Another interesting observation as can be seen in the Fig.2 (b) and 3(b) is clustering of the grains in the shape of rings and the increasing grain density in each cluster with increasing temperature. This might be due to the presence of boron doping during high temperature annealing of the PS film. It has been analysed that the electrical conductivity of the PS film is controlled by the boron doping profile and does not vary due to grains or clustering of the grains. Also boron has a tendency of diffusion inside the grains. The ordered clustering of the PS grains seems attractive as it leads to nano-scale entity which could be realized by reducing the grain size during LPCVD. The present work advocates a doping specie dependent phenomenon at annealing temperatures in the LPCVD PS film resulting in to stabilize grain size with associated ordered clustering of the grains which grows further with increasing temperature.

Experimental observation of aligning of the grains in a shape of ring like cluster has been shown in LPCVD PS film on Silicon nitride. Temperature dependent growth of the PS rings with no change in the grain size and stipend conductivity provides a new front in the area of nanotechnology. The technology seems to BE COMPETENT TO REALIZE NANOSCALE STRUCTURES IN SOI ENVIRONMENT.

\section{Acknowledgement}

Thanks for School of Physical Sciences, JNU for AFM experiment. Thanks for University Grants Commission, Kathmandu, for fellowship to the Author.

\section{References}

Tilke, A., R.H. Blick, H. Lorenz and J.P. Kotthaus. 2002. Single-electron effects in highly doped polysilicon nanowires. Physica E. low dimensional system and nanostructures 15(2): 60-64.

McConnel, A.D., S. Uma. E. Goodson. 2001. Thermal Conductivity of Doped Polysilicon Layers. IEEE/ ASME Journal of MicroElectro Mechanical Systems, 10: $360-369$

Tai, Y.C., C.H. Mastrangelo and R.S. Muller. 1988. Thermal conductivity of heavily doped low-pressure chemical vapor deposited polycrystalline silicon films", J. Appl. Phys. 63: 950.

Chang, C.Y., and S.M. Sze. (Eds.) 1996. VLSI Technology", McGraw-Hill, New York.

Maseeh, F., and S.D. Senturia 1990. "Plastic Deformation of Highly Doped Silicon", Sensors and Actuators. A21 - A23: 861-865.

Kalainathan, S., R. Dhanasekaran and P. Ramasamy. 1990. Grain size and size distribution in heavily phosphorus doped polycrystalline silicon. Journal of Crystal Growth 104: 250-256.

Franco, G., M. Priulla, G. Renna and G. Scerra. 2001 Influence of the polysilicon doping on the electrical quality of thin oxides: a confrontation between vertical and horizontal furnaces. Materials Science in Semiconductor Processing. 4 (1-3): 153-157

Karnik, Sooraj V. and Miltiadis K. Hatalis 2003. Lateral polysilicon $\mathrm{p}^{+}-\mathrm{p}-\mathrm{n}^{+}$and $\mathrm{p}^{+}-\mathrm{n}-\mathrm{n}^{+}$diodes", Solid-State Electronics 47 (0-4) 653-659

Pacellia, A., S. Spinelli and L.M. Perron. 1999. Carrier quantization at flat bands in MOS devices. IEEE Transactions on Electron Devices 46 (2): 383-387

Eccleston, W., 1999. The effect of polysilicon grain boundaries on MOS based devices. Microelectronic Engineering 48: $105-108$

Ahmad, I., A. Omar, A. Hussain and A. Mikdad 2002. Characterization of polysilicon gate microstructures for $0.5 \mathrm{~mm}$ CMOS devices using transmission electron microscopy and atomic force microscopy images. Applied Surface Science 191: 362-367

Guinier, A., R. Gullien. 1989. The solod state to superalloys; International union of crystallography. Oxford university press. 
Nepal Journal of Science and Technology 10 (2009) 